\title{
A modified time-of-flight (TOF) method for precise determination of high speed ratios in molecular beams
}

\author{
A. Salvador Palau, ${ }^{1}$ S.D. Eder, ${ }^{1,}$ a) T. Kaltenbacher, ${ }^{1}$ B. Samelin, ${ }^{1}$ G. Bracco, ${ }^{1,2}$ and B. Holst ${ }^{1}$ \\ 1) Department of Physics and Technology, University of Bergen, Allégaten 55, 5007 Bergen, \\ Norway \\ ${ }^{2)}$ CNR-IMEM, Department of Physics, University of Genova, V Dodecaneso 33, 16146 Genova, \\ Italy
}

(Dated: 12 January 2016)

Time-of-flight (TOF) is a standard experimental technique for determining, among others, the speed ratio $S$ (velocity spread) of a molecular beam. The speed ratio is a measure for the monochromaticity of the beam and an accurate determination of $S$ is crucial for various applications, for example for characterising chromatic aberrations in focussing experiments related to helium microscopy or for precise measurements of surface phonons and surface structures in molecular beam scattering experiments. For both of these applications it is desirable to have as high a speed ratio as possible. Molecular beam TOF measurements are typically performed by chopping the beam using a rotating chopper with one or more slit openings. The TOF spectra are evaluated using a standard deconvolution method. However, for higher speed ratios, this method is very sensitive to errors related to the determination of the slit width and the beam diameter. The exact sensitivity depends on the beam diameter, the number of slits, the chopper radius and the chopper rotation frequency. We present a modified method suitable for the evaluation of TOF measurements of high speed ratio beams. The modified method is based on a systematic variation of the chopper convolution parameters so that a set of independent measurements that can be fitted with an appropriate function are obtained. We show that with this modified method it is possible to reduce the error by typically one order of magnitude compared to the standard method.

Keywords: time-of-flight, molecular beams, speed ratio

\section{INTRODUCTION}

The velocity spread of a molecular beam and hence its monochromaticity is an important parameter for applications such as neutral atom beam microscopy ${ }^{1-7}$ and molecular beam scattering experiments ${ }^{8,9}$. In many of these applications a narrow velocity distribution is important and it is important also to be able to determine the width of the distribution precisely. The molecular beams used are typically so-called thermal beams, created in a supersonic expansion (free-jet expansion). The beam expands from a high pressure reservoir through a small nozzle into vacuum and the central part of the beam is selected by a skimmer. The velocity distribution of such a beam along the beam axis can be described by a Maxwell Boltzmann distribution ${ }^{10,11}$ which for high $\mathrm{S}$ values can be well approximated with a Gaussian distribution. The velocity distribution for a thermal molecular beam is usually expressed in terms of the speed ratio $S=2 \sqrt{\ln (2)} v / \triangle v$, where $v$ is the average beam velocity and $\triangle v$ is the full width at half maximum (FWHM) of the (approximately Gaussian) velocity distribution ${ }^{10,12,13}$. The most common experimental method used to determine the kinetic energy of such beams is time-of-flight ${ }^{14-16}$. Here, the beam is pulsed using a chopper with one or more slit openings and the

a) Corresponding author, sabrina.eder@uib.no; The two first authors contributed equally to this work time $t$ required for the atoms or molecules in each pulse to travel a certain distance $L$ is measured. Two types of choppers are commonly used, the so called pseudorandom chopper, which enables a high beam transmissivity, but is not suited for measuring narrow velocity distributions ${ }^{17-19}$ and a "standard" chopper, which has one or more rectangular slits of the same size and equidistantly spaced around the chopper periphery. For a standard chopper the measured time distribution can be converted into a beam velocity distribution ${ }^{20}$ using the standard deconvolution method presented by Pauly ${ }^{10}$. However for high speed ratios this method leads to a large uncertainty, how large depends on the slit width and chopper frequency. For our set-up the uncertainty increases to $\approx 5 \%$ for speed ratios above 140 . In this paper we present a modified evaluation method for the time distribution based on doing several measurements with a trapezoidally shaped slit adjusted to different heights. The paper begins with a short description of how TOF spectra are obtained experimentally, followed by an evaluation of the standard deconvolution method, showing its limitation for high speed ratios. Then comes a presentation of our new, modified method including the experimental requirements and a comparison of the new and the standard method.

\section{EXPERIMENTAL SET-UP FOR TOF MEASUREMENTS}

Fig. 1 shows the TOF measurement set-up used to obtain the experimental measurements presented here. It 
is a typical set-up, only that we are using trapezoidal rather than rectangular slits and that for surface science experiments the beam will reflect off a surface before or after chopping. All measurements have been obtained using the molecular beam apparatus at the University of Bergen, popularly known as MAGIE. A detailed instrument description can be found in ${ }^{21}$. During a TOF experiment the chopper, which can have several slit openings, rotates with a certain frequency $f_{\mathrm{ch}}$, chopping the beam into short pulses (sometimes referred to in the literature as beam packages). A drawing of the chopper disc used here is shown in Fig. 2. A detailed drawing of a trapezoidal slit is shown in Fig. 3. In addition to two trapezoidal slits, the chopper disc has two small rectangular slits placed above the centre of each trapezoidal slit. They are used to provide a trigger signal for a flight time measurement of a beam pulse. This trigger pulse is generated by a light-emitting-diode (LED) and a photo-detector, mounted on each side of the chopper wheel. The 10 small slits are used to control the rotation frequency. The 10 large slits present a 50\% duty cycle and can be used to modulate the incident beam for measurements of elastic diffraction, detecting the signal by means of a lock-in amplifier. These slits were not used in these experiments. The slit width is changed by manually changing the position of the chopper relative to the beam. This can be done with micron precision using a translation stage. At the end of the beam line the atoms are detected using an ionization detector. After a preset delay time $t_{\mathrm{d}}$, the TOF electronics starts to count the number of detected atoms in predefined time-bins. To gain enough signal intensity, a TOF spectrum is integrated over thousands of beam pulses. Fig. 4 shows a measured time distribution with a single Gaussian fit.

\section{THE STANDARD TOF DECONVOLUTION METHOD}

The mean flight time $t_{\mathrm{f}}$ of the beam atoms can be obtained from the TOF spectrum as follows:

$$
t_{\mathrm{f}}=t_{\mathrm{d}}+t_{\mathrm{p}}-t_{\mathrm{c}}-t_{\mathrm{ch}} / 2
$$

where $t_{\mathrm{d}}$ is the pre-set delay time (see previous section). $t_{\mathrm{p}}$ the peak time extracted from the measured spectrum, $t_{\text {ch }}$ the set channel width of the recorded signal parts (time bin) (see previous section) and $t_{c}$ a correction factor caused by the positioning of the trigger slit relative to the beam chopping slit. This correction factor is inversely proportional to $f_{c h}$ and can be determined experimentally using a calibration procedure ${ }^{22}$. Using the flight distance $L$, the average velocity of the beam is thus found to be $v_{\mathrm{f}}=L / t_{\mathrm{f}}$.

To convert a TOF time distribution spectrum into a velocity distribution spectrum it has to be taken into account that the true time distribution spectrum of the beam is perturbed in the data taking process. Firstly each beam pulse has a certain duration determined by the slit width and rotation frequency. The relative movement of the atoms within a beam pulse cannot be measured. Secondly, the ionization detection leads to an error. The filament has a finite extension and it cannot be distinguished if an atom was ionized at the beginning of the filament or at the end.

In the standard TOF evaluation method it is assumed that these two contributions lead to Gaussian broadenings of the approximately Gaussian velocity distribution of the beam. The final TOF spectrum is taken to be a convolution of three independent Gaussian distributions ${ }^{14}$. One can thus obtain a relation between the FWHM of the distributions:

$$
\Delta t_{\mathrm{M}}^{2}=\Delta t_{\mathrm{v}}^{2}+\Delta t_{\mathrm{C}}^{2}+\Delta t_{\mathrm{D}}^{2}
$$

where $\Delta t_{\mathrm{M}}$ is the FWHM of the measured distribution. $\Delta t_{\mathrm{v}}$ is the FWHM of the real beam time distribution, $\Delta t_{\mathrm{C}}$ the FWHM of the contribution due to the finite pulse width and $\Delta t_{\mathrm{D}}$ is the FWHM of the contribution due to the filament of the ionizer. According to Pauly ${ }^{14}$ Equation (2) can be re-written as follows

$$
\Delta t_{\mathrm{v}}^{2}=\left(L \cdot \frac{\Delta v}{v_{\mathrm{f}}^{2}}\right)^{2}=\Delta t_{\mathrm{M}}^{2}-\left(\frac{o}{2 \pi r_{\mathrm{ch}} f_{\mathrm{ch}}}\right)^{2}-\left(\frac{L_{\mathrm{D}}}{v_{\mathrm{f}}}\right)^{2}
$$

with $L_{\mathrm{D}}$ the detector ionization length, $r_{\mathrm{ch}}$ the chopper disc radius at the centre of the beam, $f_{\mathrm{ch}}$ the chopper frequency and the opening, $o$, is defined as follows:

$$
o= \begin{cases}w & w \geq 2 b \\ 2 b & w<2 b\end{cases}
$$

where $w$ is the slit width and $\mathrm{b}$ is the beam radius ${ }^{14}$. $\mathrm{S}$ can be rewritten as:

$$
S=2 \sqrt{\ln 2} \frac{v_{\mathrm{f}}}{\Delta v}=2 \sqrt{\ln 2} \frac{t_{\mathrm{f}}}{\Delta t_{\mathrm{v}}}\left(1-\left(\frac{\Delta t_{\mathrm{v}}}{2 t_{\mathrm{f}}}\right)^{2}\right) .
$$

If $t_{\mathrm{f}}>>\frac{\Delta t_{\mathrm{v}}}{2}$ we can approximate equation (4) to

$$
S \approx 2 \sqrt{\ln 2} \frac{t_{\mathrm{f}}}{\Delta t_{\mathrm{v}}}
$$

Combining equations (3) and (5) the deconvoluted speed ratio $S_{\mathrm{de}}$, corresponding to the real speed ratio of the atom beam, can be obtained:

$$
S_{\mathrm{de}} \approx 2 \sqrt{\ln 2} \frac{t_{\mathrm{f}}}{\sqrt{\Delta t_{\mathrm{M}}^{2}-\left(\frac{o}{2 \pi r_{\mathrm{ch}} f_{\mathrm{ch}}}\right)^{2}-\left(\frac{L_{\mathrm{D}}}{v_{\mathrm{f}}}\right)^{2}}},
$$

from the Gaussian error propagation of (6) follows

$$
\begin{aligned}
& \sigma_{S_{d e}}^{2}=\text { R.O.T }+\ldots \\
& \quad 4 \ln 2 \frac{t_{\mathrm{f}}^{2}}{\Delta t^{6}}\left(\frac{o^{2} r_{\mathrm{ch}}^{\prime}(o)}{\left(2 \pi f_{\mathrm{ch}}\right)^{2} r_{\mathrm{ch}}^{3}(o)}-\frac{o}{\left(2 \pi f_{\mathrm{ch}}\right)^{2} r_{\mathrm{ch}}^{2}(o)}\right)^{2} .
\end{aligned}
$$

Where for large values of $\left(\Delta t_{\mathrm{C}}=\frac{o}{2 \pi r_{\mathrm{ch}} f_{\mathrm{ch}}}\right)$, and high speed ratios, an error associated to the opening $o$ and the chopper radius $r_{\mathrm{ch}}$ dominates the obtained speed ratio $S_{\text {de }}$ (see Fig.5). 


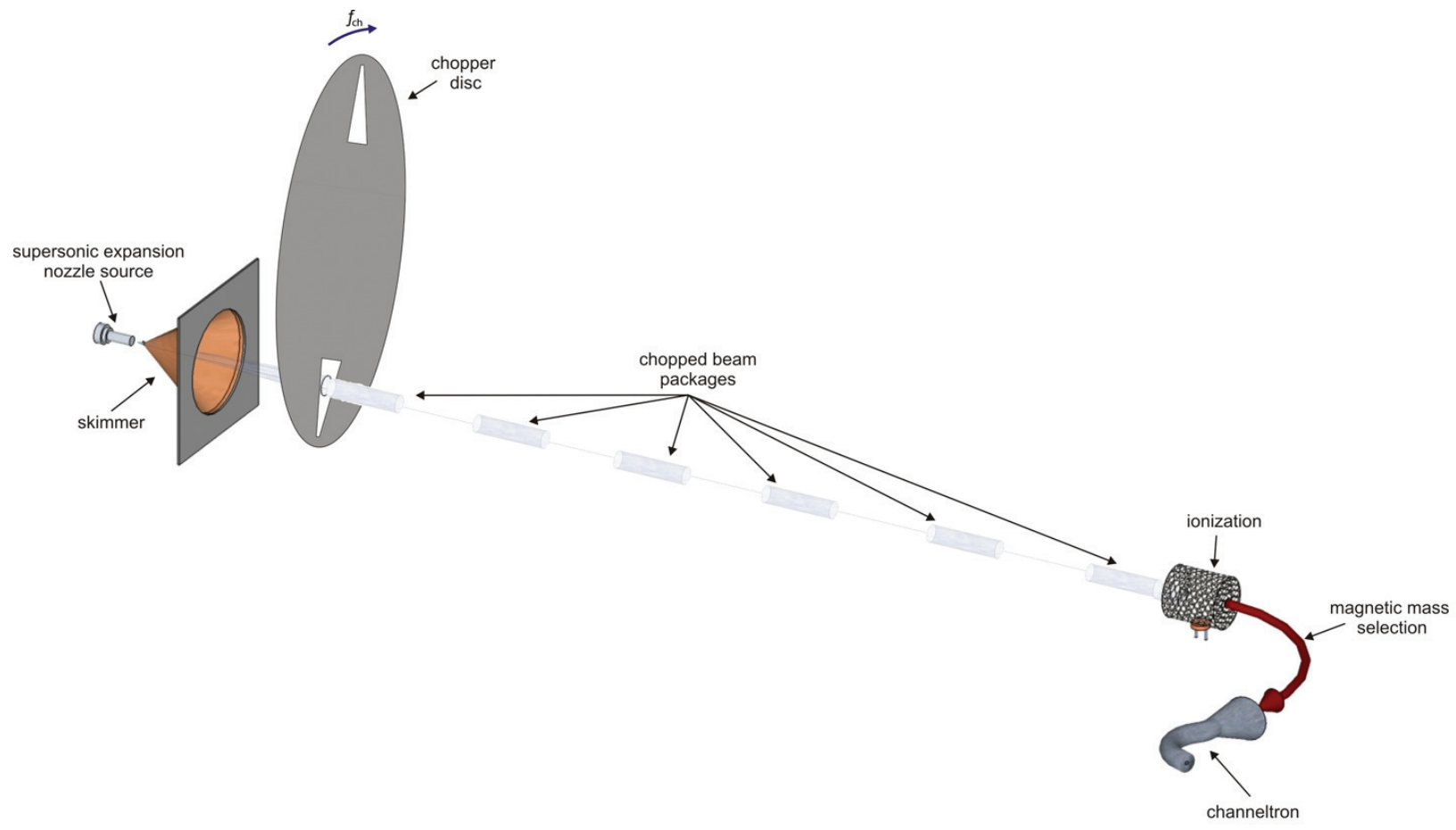

FIG. 1. Diagram of the TOF set-up on MAGIE. A chopper disc is placed in the neutral helium beam line, creating well defined beam pulses (beam packages) which are detected in an ionization detector.

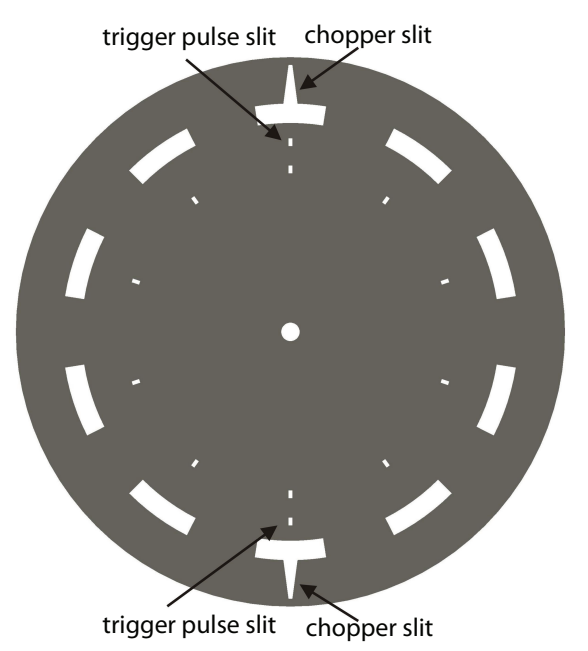

FIG. 2. Drawing of the chopper disc used in the experiments presented here. Two trapezoidal slits for beam chopping are placed opposite to each other. Two small rectangular slits centered above the trapezoidal slits are used for generating trigger pulses to the measurement electronics. The 10 small slits are used in a similar manner for generating trigger pulses to control the rotating frequency. The large slit openings are not used here.

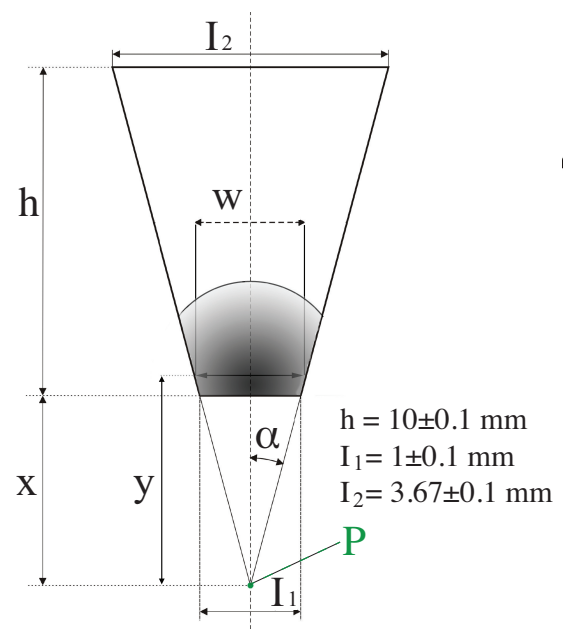

FIG. 3. Diagram of a trapezoidal slit as used here. Point $\boldsymbol{P}$ is the extrapolated tip position. The shaded area illustrates how the beam crosses the slit, darker tones correspond to a higher beam intensity. $x$ indicates the distance from the extrapolated tip position to the actual end of the slit. $y$ indicates the distance from the extrapolated tip position to the center of the beam. 


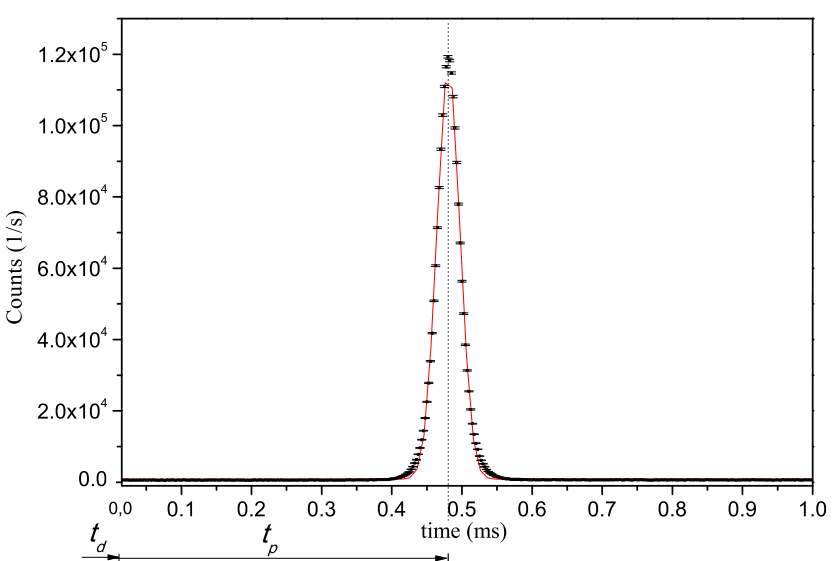

FIG. 4. TOF spectrum of a free-jet helium expansion beam from a $10 \mu \mathrm{m}$ nozzle with a stagnation temperature and pressure of $T_{0}=295 \mathrm{~K}$ and $p_{0}=81$ bar. $t_{\mathrm{d}}$ is a predefined delay time following the trigger pulse, preceding the actual measurement. The accumulated signal from the incoming helium atoms is then recorded in certain predefined time-bins with increasing time-line. The peak time $t_{\mathrm{p}}$ is defined by the peak position of the spectrum. The fitting is performed to estimate the FWHM.

\section{THE MODIFIED TOF EVALUATION METHOD}

\section{General idea}

An optimum opening $o$ would be indefinitely small, but this is clearly not realisable. With our evaluation method we utilize the chopper slit's trapezoidal shape to obtain a span of measurements for different slit widths $w$. The same procedure could also be implemented using a rectangular slit but the variation in the measured values would be smaller. Alternatively, independent measurements could be achieved by varying any of the physical parameters affecting the convolution, such as the chopper frequency or the beam radius. By fitting the gained curve with a given analytical function, where one of the fitting parameters is the FWHM of the real velocity spread $\left(\Delta t_{\mathrm{v}}\right)$, we are able to obtain the deconvoluted speed ratio with a much smaller associated uncertainty. The method is divided in two parts, first there is a spatial calibration of the size of the beam and width of the slit at every point. Second, the data is fitted with the analytical function described in section IV 3.

\section{Spatial Calibration}

The methods used to determine the convoluted speed ratio depend strongly on the beam radius $b$ and the chopper slit width $w$. Normally they are inferred on the basis of independent measurements of the slit width, combined with knowledge about how the beam has been collimated. However, a more precise value can be obtained from fit-

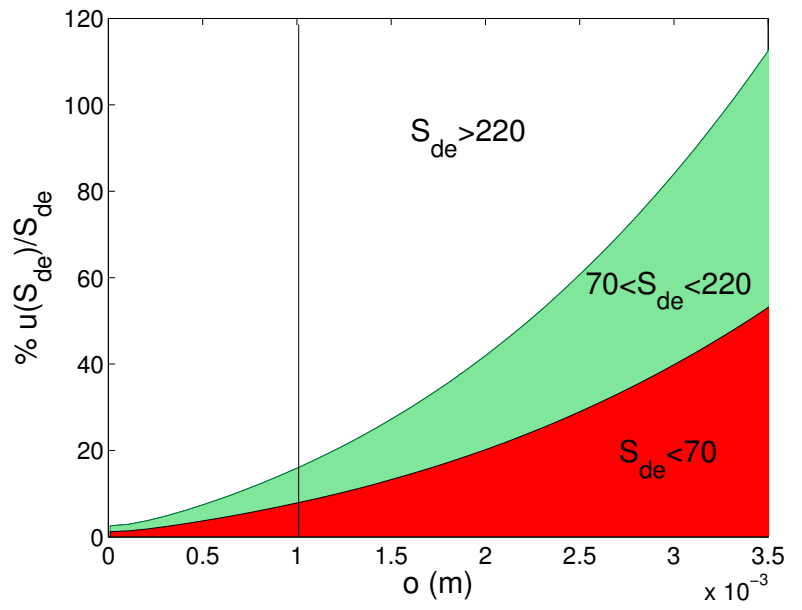

FIG. 5. Uncertainty of $S_{\mathrm{de}}, \% u\left(S_{\mathrm{de}}\right) / S_{\mathrm{de}}$ versus $o$ for different deconvoluted speed ratios. The S-isometric curves show a monotone increase of the uncertainty with the opening, $o$ and the deconvoluted speed ratio. The associated error in $o$ was chosen to be $\Delta o \approx 0.1 \mathrm{~mm}$. The minimum value of $o$ achievable with our system is given by the minimum slit width, marked with a vertical black line. The rest of the physical parameters are set according to the experimental set-up; $f_{\mathrm{ch}}=310 \mathrm{~Hz}, r_{\mathrm{ch}}=0.0727 \mathrm{~m}-3.7453 w$ (for the case $w>2 b$, each slit width corresponds to a slightly different chopper radius).

ting the measured intensity values of the beam on its descent out of the trapezoidal chopper slit,

$$
I(w)=\mu+K \cdot T\left(w, f_{\mathrm{ch}}\right) \cdot A(w) \cdot E(w),
$$

where $T\left(w, f_{\mathrm{ch}}\right)$ is the time opening for a slit width $w$ at a given chopper frequency $f_{\text {ch }} . A(w)$ is the cross section of the beam as seen in Fig. 3. $E(w)$ is the integral of the normalized beam distribution along the $y$ axis after intersecting the slit, this integral is computed using an error function (see equation (10)). $K$ is the total intensity of the beam at the chopper disk position in counts/second and $\mu$ is the background count rate when the chopper is completely blocking the beam. The contributions are given as follows:

$$
T\left(w, f_{\mathrm{ch}}\right)=\frac{w}{\pi f_{\mathrm{ch}} r_{\mathrm{ch}}},
$$

where $r_{\text {ch }}$ is the radius of the rotating chopper disk leading to slit width $w$.

$$
E(w)=\frac{1}{2}(1+\operatorname{erf}(\delta))
$$

where

$$
\delta=\frac{(y-x) \sqrt{\ln 2}}{b},
$$

where $y$ is the distance from $\mathbf{P}$ to the centre of the beam and $x$ the distance from $\mathbf{P}$ to the end of the slit (see Fig. 
3). The distance $y$ can be related to $w$ by means of the angle $\alpha, y=\frac{w}{2 \tan \alpha}$, and $b$ is the beam radius. As illustrated in Fig. $6, A(w)$ must be calculated according to the geometrical bounds of the problem. Heaviside functions are used to express all possible domains within a single equation (see the Appendix).

$$
\begin{gathered}
A(w)= \\
H_{s}(\pi-\gamma)\left(1-H_{s}\left(h^{\prime}\right)\left\{\frac{h^{\prime}}{2 \pi b^{2}}\left(I_{1}+2 b \sin (\beta-\alpha)\right)\right.\right. \\
\left.\left.-\frac{1}{2 \pi}(2 \beta-2 \alpha-\sin (2 \beta-2 \alpha))\right\}-\frac{1}{\pi}(\theta-\sin \theta)-\frac{1}{2 \pi}(\gamma-\sin \gamma)\right) \\
+H_{s}(\gamma-\pi) \frac{1}{2 \pi}(\gamma-\sin \gamma), \quad(12)
\end{gathered}
$$

with

$$
h^{\prime}=x-y+b \cos (\beta-\alpha) .
$$

$H_{\mathrm{s}}$ is the heaviside function. The angles $\alpha, \beta, \gamma$ and $\theta$ are defined in Fig. 6.

The spatial calibration described in (8) has three fitting parameters: the relative beam position $y-x$, which is proportional to the slit width $w$, the beam radius $b$ and the intensity constant $K$. The initial fit parameters are chosen as follows: $K_{0}$ is obtained by fitting equation (8) in the range where the influence of the beam extinction is negligible i.e., $A(w) \approx E(w) \approx 1 . b_{0}$ is set at $0.5 \mathrm{~mm}$ for a nozzle diameter of $10 \mu \mathrm{m}$ and a skimmer diameter of $4 \mu \mathrm{m}$. The initial beam height relative to the slit is obtained by measuring the beam intensity drop as the chopper is moved manually across the beam. At the point where the intensity reaches the minimum, the beam is out of the slit. All the aforementioned parameters are varied within the spatial calibration to determine the best fit for the measured beam intensity values (see Fig. 7).

The described calibration can be improved by assuming an axisymmetric Gaussian distribution of the beam intensity and performing a numerical convolution with the trapezoidal slit and the skimmer. However, equation (8) achieves good parametric precisions due to the fact that all geometrical domains are considered (see equation (12)).

\section{Fitting function}

The standard deconvolution method described in section III models the transmission function as a Gaussian with $\Delta t_{\mathrm{C}}$ as its FWHM:

$$
S_{\mathrm{C}}=\frac{2 \sqrt{\ln 2} \cdot t_{\mathrm{f}}}{\sqrt{\Delta t_{\mathrm{v}}^{2}+\Delta t_{\mathrm{C}}(y)^{2}+\Delta t_{\mathrm{D}}^{2}}},
$$

with $\Delta t_{\mathrm{C}}=\frac{o}{2 \pi r_{\mathrm{ch}} f_{\mathrm{ch}}}$ and $\Delta t_{\mathrm{D}}=\frac{L_{\mathrm{D}}}{v_{\mathrm{f}}}$. Tests have shown that this equation does not fit our data set well (see Fig.

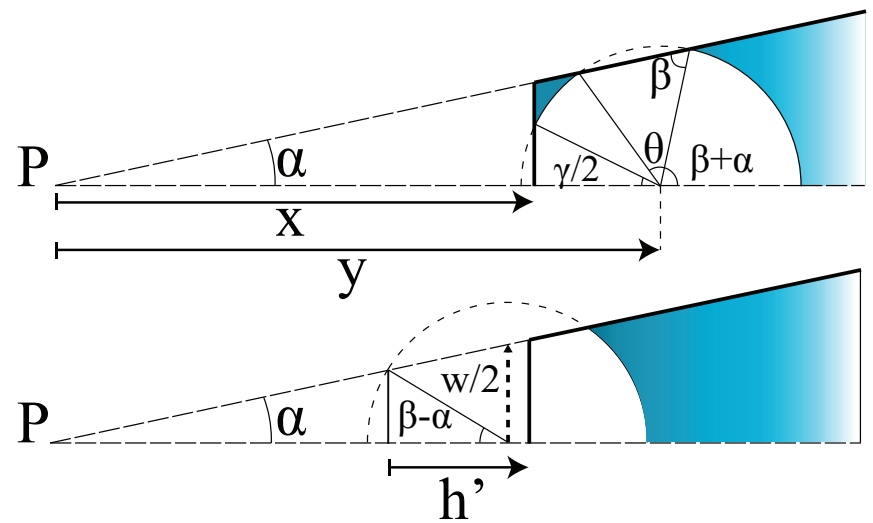

FIG. 6. Side view of the chopper slit with the angles used in the equation (12) for $A(w)$. The line corresponding to the end of the slit is marked with a darker color.

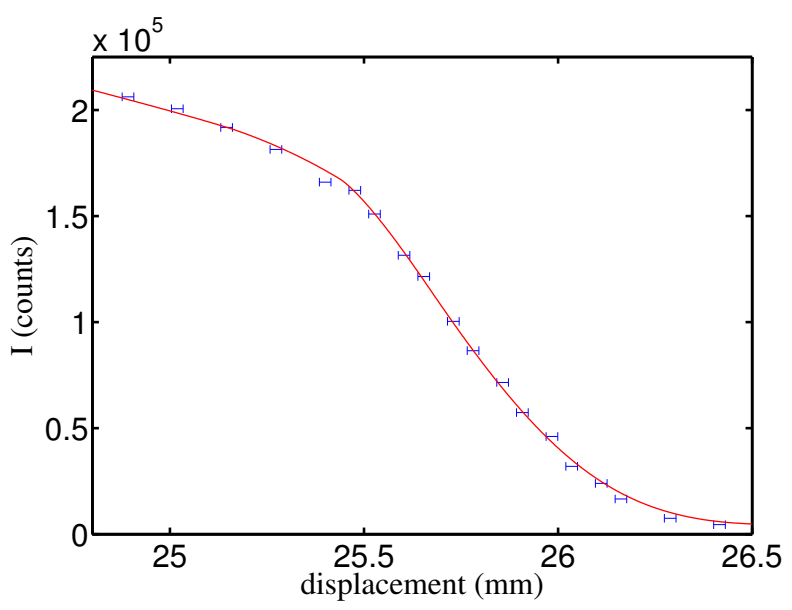

FIG. 7. Spatial calibration. The measured beam intensity (blue points) is plotted against the chopper disc displacement. For a beam stagnation pressure po $=90 \mathrm{bar}, f_{\mathrm{ch}} \approx 229 \mathrm{~Hz}$, and a beam temperature of $T_{0}=125 K$. The red line shows the fitted spatial calibration described in equation (8). From the fit the slit width for each point and beam radius are determined, in this case $b=0.57 \pm 0.01 \mathrm{~mm}$. The uncertainty of the intensity values is low, $\sigma_{I}<0.004 \cdot 10^{5}$ counts.

9 ). We have therefore introduced a new function which includes a parameter defined as area preserving sigma, $\sigma_{\mathrm{A}}$, to correct for the in reality non Gaussian transmission function. This parameter is defined as a positive real number that covers an area of the normalized choppertransmission function equivalent to the area covered by $1 \sigma$ in a Gaussian distribution.

$$
\int_{-\sigma_{\mathrm{A}}}^{\sigma_{\mathrm{A}}} T_{\mathrm{eff}} d t=0.68268
$$

where the transmission function, $T_{\text {eff }}$ is a trapezoidal function such as defined in $^{14}$. $\sigma_{\mathrm{A}}$ is used to obtain an alternative FWHM to represent the slit by $\Delta t_{\mathrm{CA}}=$ $2 \sqrt{2 \ln 2} \sigma_{\mathrm{A}}$. However, this new parameter, when used alone, still does not provide a satisfactory fit. A well- 
fitting function can be obtained by averaging both parameters (see Fig. 9);

$$
\Delta t_{\text {Cavg }}=\frac{1}{2}\left(\Delta t_{\mathrm{C}}+\Delta t_{\mathrm{CA}}\right)
$$

where $\Delta t_{\text {Cavg }}$ is the modified FWHM resulting from the average of $\Delta t_{\mathrm{C}}$ and $\Delta t_{\mathrm{CA}}$. The convoluted speed ratio values, $S_{\mathrm{C}}$ are thus obtained as:

$$
S_{\mathrm{C}}=\frac{2 \sqrt{\ln 2} \cdot t_{\mathrm{f}}}{\sqrt{\Delta t_{\mathrm{v}}^{2}+\Delta t_{\text {Cavg }}(y)^{2}+\Delta t_{\mathrm{D}}^{2}}},
$$

where the flight time $t_{\mathrm{f}}$ is calculated using equation (1), $\Delta t_{\mathrm{v}}$ is the fitting parameter. $\Delta t_{\text {Cavg }}$ is implicitly dependent of $y$, which is the height at which the slit is situated for each of the measured $S_{\mathrm{C}}$ values with $y=0$ defined at the point where the center of the beam crosses the end of the slit. The deconvoluted value of $S$ is then obtained using equation 5 by setting $\Delta t$ to the obtained value $\Delta t_{\mathrm{v}}$ from the fitting function. The uncertainty calculation is performed by weighting every measured speed ratio point by $1 / \sigma_{\mathrm{p}}^{2}$ where $\sigma_{\mathrm{p}}$ accounts for its standard deviation, obtained by a Gaussian uncertainty propagation. As can be clearly seen in Fig. 8, the uncertainty values for the final speed ratio $S_{\mathrm{de}}$ obtained by the presented method are significantly smaller than the uncertainty values obtained using the standard deconvolution method described in section III.

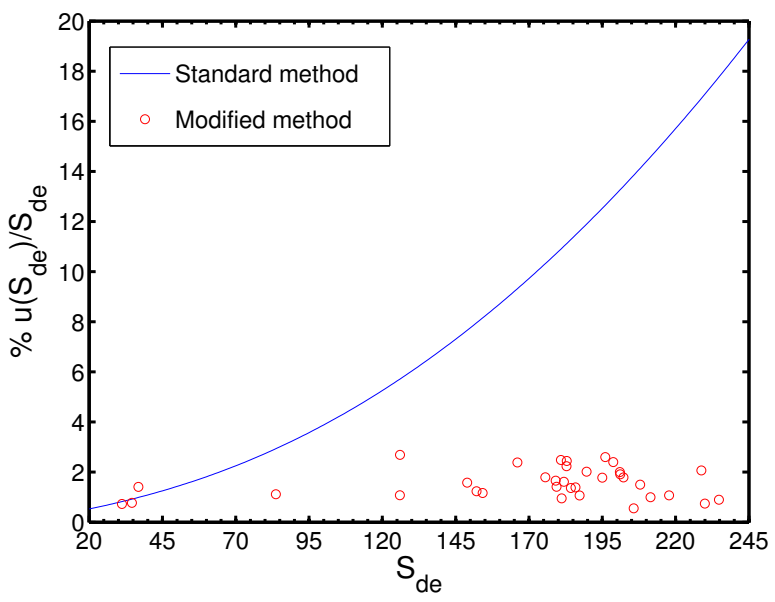

FIG. 8. Uncertainties for the deconvoluted speed ratio values, $S_{d e}$. The blue line plots the minimum uncertainty values obtainable using the standard deconvolution method, corresponding to the values over the black line at Fig. $5(o=1 \mathrm{~mm})$. The red circles are the uncertainty values obtained under the same conditions using the modified deconvolution method. The red circles are scattered due to their direct dependence on the statistical goodness for each experiment.

\section{CONCLUSION}

We have presented a high precision method to evaluate the speed ratio of a thermal molecular beam for a wide range of speed ratio values. The method is based on measuring the speed ratio of the beam at a range of convolution parameters, here realised by varying the chopper slit widths and fitting the data by a specially developed convoluted speed ratio function. The final speed ratio is found using the fitting parameter, which corresponds to the FWHM of the deconvoluted velocity distribution.

Furthermore, we introduce an equation for the beam intensity through the slit, which is used to fit the beam width and the position of the beam center relative to the chopper slit.

An important quality of the presented measurement method is, that it is not limited to the analytical convolution function presented in this paper. Alternative numerical or analytical models can be implemented to model the convoluted speed ratio. The decrease of the measurement uncertainty follows from the statistical redundancy of using a range of convoluted $\mathrm{S}$ values. The magnitude of this decrease will depend on the validity of the convolution model used to fit the experimental data. However, the increase of the measurement accuracy does not stem from the aforementioned statistical redundancy but solely from the soundness of the equations modelling the convolution of the beam with the slit.

\section{ACKNOWLEDGMENTS}

The work presented here was sponsored by the European Union: Theme NMP.2012.1.4-3 Grant no. 309672, project NEMI (Neutral Microscopy).

We thank the mechanical workshop of the CNR-IMEM and INFN, Department of Physics, University of Genova, Italy for building the chopper disc used in the experiment.

We also thank Truls Andersen for performing TOF measurements and contributing with datasets.

\section{APPENDIX: DERIVATION OF $E(w)$ (EQUATION (10)) AND $A(w)$ (EQUATION (12))}

The basic expression of $E(w)$ (equation (10)) is given as follows;

$$
\begin{aligned}
E(w)=\frac{1}{\sqrt{2 \pi} \sigma} & \int_{-\infty}^{y-x} e^{-\frac{\xi^{2}}{2 \sigma^{2}}} d \xi \\
& =\frac{1}{2}\left(1+\frac{2}{\sqrt{2 \pi} \sigma} \int_{0}^{y-x} e^{-\frac{\xi^{2}}{2 \sigma^{2}}} d \xi\right),
\end{aligned}
$$

where $y-x$ is the distance from the centre of the beam to the end of the slit (see Fig. 3). In order to obtain the error function, we perform the variable change $\frac{\xi^{2}}{2 \sigma^{2}}=$ 


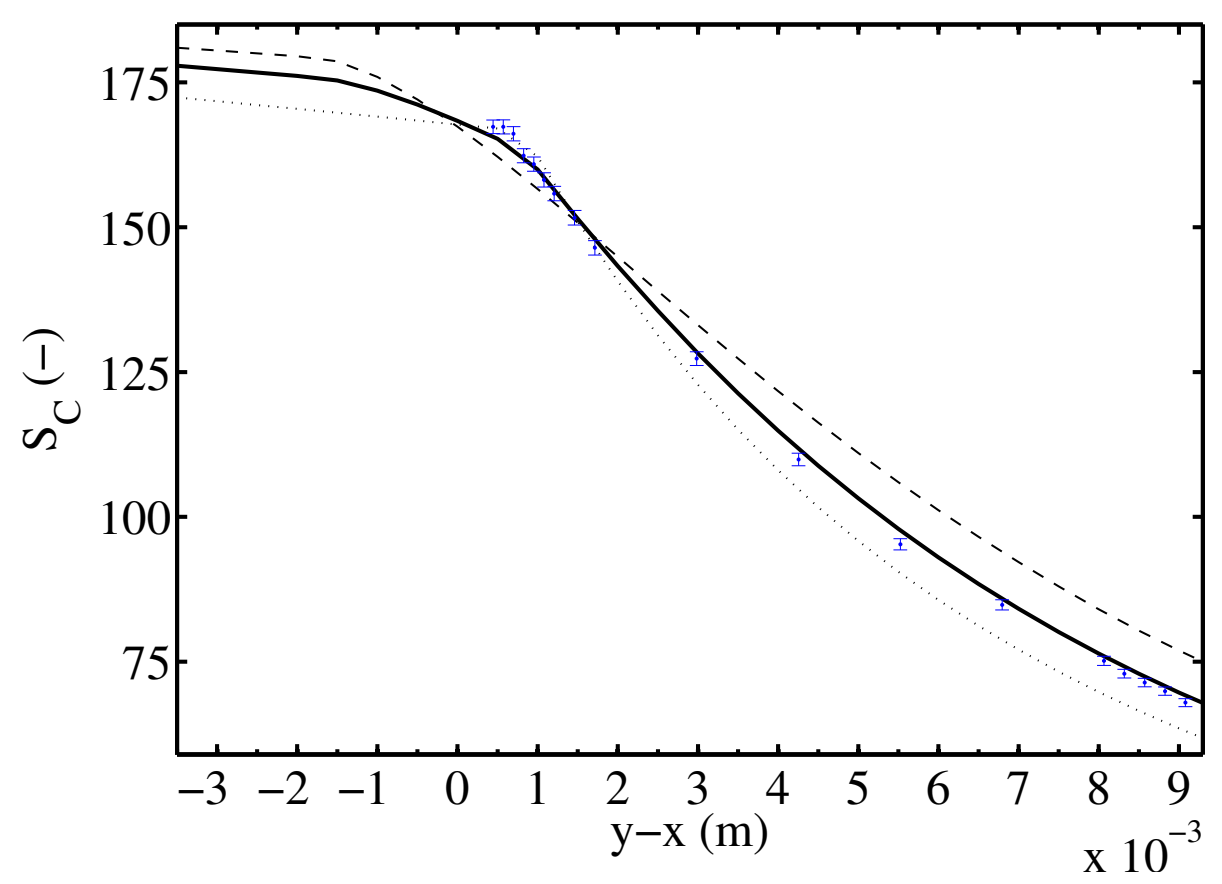

FIG. 9. Convoluted speed ratio values $S_{\mathrm{C}}$ versus the slit height (see Fig. 6) for $S_{\mathrm{de}}=181.9 \pm 2.9$. The dotted line represents equation (14). The dashed lines uses the area fitting function alone and the solid line is equation (17)

$t^{2} \rightarrow d \xi=\frac{2 \sigma}{\sqrt{2}} d t$. The beam radius $b$ is defined as half of the FWHM of the Gaussian distribution modelling the intensity profile of the beam, $b=\sqrt{2 \ln 2} \sigma$.

$$
\begin{aligned}
E(w) & =\frac{1}{2}\left(1+\frac{4 \sigma}{\sqrt{4 \pi} \sigma} \int_{0}^{\frac{y-x}{\sqrt{2} \sigma}} e^{-t^{2}} d t\right) \\
= & \frac{1}{2}\left(1+\frac{2}{\sqrt{\pi}} \int_{0}^{\delta} e^{-t^{2}} d t\right)=\frac{1}{2}(1+\operatorname{erf}(\delta)),
\end{aligned}
$$

where $\delta=\frac{(y-x) \sqrt{\ln 2}}{b}$. The error function can give positive and negative values, so equation (19) can be used for any $y-x$.

The derivation of equation (12) is more lengthy because many geometric regimes and sub-regimes must be considered. The possibilities are sketched in Fig. 10. The area resulting from intersections of this kind can be calculated by removing from the area of the circle the portions of the circle that are left outside. Or, in some cases, to directly calculate the portion that is left inside. Therefore, it is enough to use the formula for the area of a circular segment together with the area of a trapezoid (see Fig. 11). The area of the blue segment is

$$
A_{\mathrm{S}}=\frac{1}{2} b^{2}\left(\theta^{\prime}-\sin \theta^{\prime}\right)
$$

and the area of the grey trapezoid:

$$
A_{\mathrm{T}}=\frac{1}{2}(m+n) h^{\prime}
$$

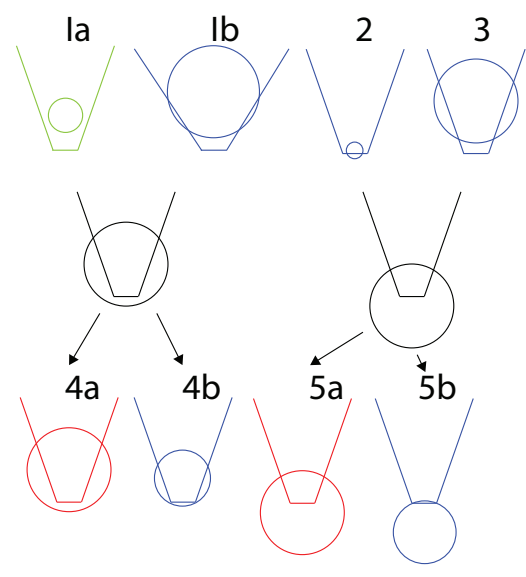

FIG. 10. The different possible geometrical cases for the intersection of a circular profile and a trapezoidal slit. In blue the cases which only require the use of equation (20) and in red the cases where equation (21) should also be applied.

It follows from Fig. 10 that to calculate the area function $A(w)$ one just needs to consider all the different cases. From Fig. $6, n=I_{1}, m=2 b \sin (\beta-\alpha)$. The angle $\theta^{\prime}$ will take different values depending on the geometrical case. The different values of $A(w)$ corresponding to each geometrical regime are included in Table I. To put all expressions in Table I within a single equation, it is useful to realise that the cases $1 \mathrm{a}, 1 \mathrm{~b}, 3,2,4 \mathrm{~b}, 4 \mathrm{a}$ and $5 \mathrm{a}$ are merely modifications of the same equation, where from the normalised area of the circle we subtract increasing 


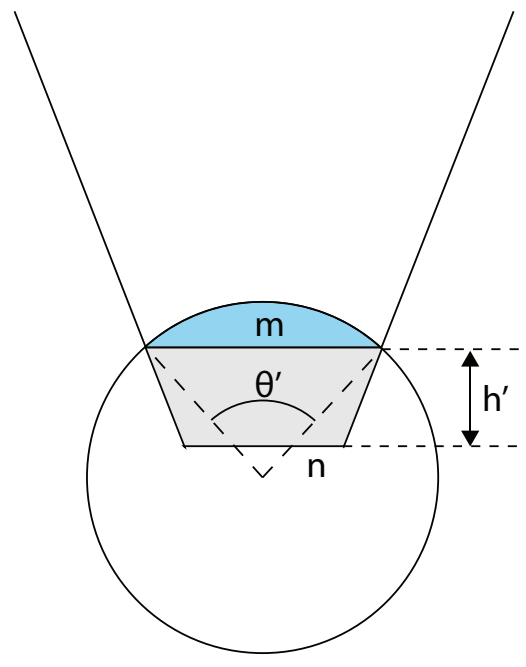

FIG. 11. Intersection of a circular profile and a trapezoidal slit. h' is the height of the trapezoid contained in the intersection, $\theta^{\prime}$ is the angle defining the circular segment and $n$ is the basis of the trapezoid.

TABLE I. Normalized area equations for all cases presented in Fig. 11 using the parameters defined in Fig. 6

\begin{tabular}{|l|l|}
\hline Case & $A(w)$ \\
\hline $1 \mathrm{a}, 1 \mathrm{~b}, 3$ & $1-\frac{1}{\pi}(\theta-\sin \theta)$ \\
\hline 2 & $1-\frac{1}{2 \pi}(\gamma-\sin \gamma)$ \\
\hline $4 \mathrm{~b}$ & $1-\frac{1}{\pi}(\theta-\sin \theta)-\frac{1}{2 \pi}(\gamma-\sin \gamma)$ \\
\hline $4 \mathrm{a}, 5 \mathrm{a}$ & \multicolumn{1}{c|}{$1-\frac{h^{\prime}}{2 \pi b^{2}}\left(I_{1}+2 b \sin (\beta-\alpha)\right)$} \\
& $-\frac{1}{2 \pi}(2 \beta-2 \alpha-\sin (2 \beta-2 \alpha))-\frac{1}{\pi}(\theta-\sin \theta)$ \\
\hline $5 \mathrm{~b}$ & $\frac{1}{2 \pi}(\gamma-\sin \gamma)$ \\
\hline
\end{tabular}

amounts of surface as the beam approaches the slit end. If $\theta, \gamma$ or $\beta$ can not be defined, they are set equal to 0 . The general solution follows,

$$
\begin{gathered}
A(w)= \\
H_{s}(\pi-\gamma)\left(1-H_{s}\left(h^{\prime}\right)\left\{\frac{h^{\prime}}{2 \pi b^{2}}\left(I_{1}+2 b \sin (\beta-\alpha)\right)\right.\right. \\
\left.\left.-\frac{1}{2 \pi}(2 \beta-2 \alpha-\sin (2 \beta-2 \alpha))\right\}-\frac{1}{\pi}(\theta-\sin \theta)-\frac{1}{2 \pi}(\gamma-\sin \gamma)\right) \\
+H_{s}(\gamma-\pi) \frac{1}{2 \pi}(\gamma-\sin \gamma) . \quad(22)
\end{gathered}
$$

${ }^{1}$ R. B. Doak, R. E. Grisenti, S. Rehbein, G. Schmahl, J. P. Toennies, and C. Wöll, Phys. Rev. Lett. 83, 4229 (1999).

${ }^{2}$ M. Koch, S. Rehbein, G. Schmahl, T. Reisinger, G. Bracco, W. E. Ernst, and B. Holst, Journal of Microscopy 229, 1 (2008).

${ }^{3}$ S. D. Eder, T. Reisinger, M. M. Greve, G. Bracco, and B. Holst, New Journal of Physics 14, 073014 (2012).

${ }^{4}$ M.-B. K.M. Martini, W. Franzen, Review of Scientific Instruments 58 (1987).

${ }^{5}$ P. Witham and E. Sanchez, Rev. Sci. Instrum 82, 103705 (2011). ${ }^{6}$ S. D. Eder, B. Samelin, G. Bracco, K. Ansperger, and B. Holst, Rev. Sci. Instrum 84 (2013).

${ }^{7}$ A. Fahy, M. Barr, J. Martens, and P. Dastoor, Rev. Sci. Instrum 86 (2015).

${ }^{8}$ D. Farias and K.-H. Rieder, Reports on Progress in Physics 61, 1575 (1998).

${ }^{9}$ B. Holst and G. Bracco, "Surface science techniques," (Springer, 2013) Chap. 12, pp. 333-367.

${ }^{10} \mathrm{H}$. Pauly, "Atom, molecule, and cluster beams 1," (Springer, 2000) Chap. 1, pp. 105-111.

${ }^{11} \mathrm{~J}$. Toennies and K. Winkelmann, The Journal of Chemical Physics 66, 3965 (1977).

${ }^{12}$ J. Braun, P. K. Day, J. P. Toennies, G. Witte, and E. Neher, Rev. Sci. Instrum 68, 3001 (1997).

${ }^{13} \mathrm{~L}$. Pedemonte and G. Bracco, The Journal of Chemical Physics 119, 1433 (2003).

${ }^{14} \mathrm{H}$. Pauly, "Atom, molecule, and cluster beams 2," (Springer, 2000) Chap. 3.2, pp. 156-171.

${ }^{15}$ D. J. Auerbach, "Atomic and molecular beam methodes," (Oxford University Press, 1988) Chap. 14, pp. 362-379.

${ }^{16}$ J. R. Buckland, R. L. Folkerts, R. B. Balsod, and W. Allison, Measurement Science and Technology 8, 933 (1997).

${ }^{17}$ R. David, K. Kern, P. Zeppenfeld, and G. Comsa, Rev. Sci. Instrum 57, 2771 (1986).

${ }^{18}$ D. D. Koleske and S. J. Sibener, Rev. Sci. Instrum 63 (1992).

${ }^{19}$ V. L. Hirschy and J. P. Aldridge, Rev. Sci. Instrum 42 (1970).

${ }^{20}$ D. Miller, "Atomic and molecular beam methodes," (Oxford University Press, 1988) Chap. 2, pp. 14-53.

${ }^{21}$ A. Apfolter, Master's thesis, Graz University of Technology (2005).

${ }^{22}$ J. L. Luneng, Low-energy surface vibration measurements on the alpha-quartz (0001) surface, Master's thesis, University of Bergen, Norway (March 2011). 